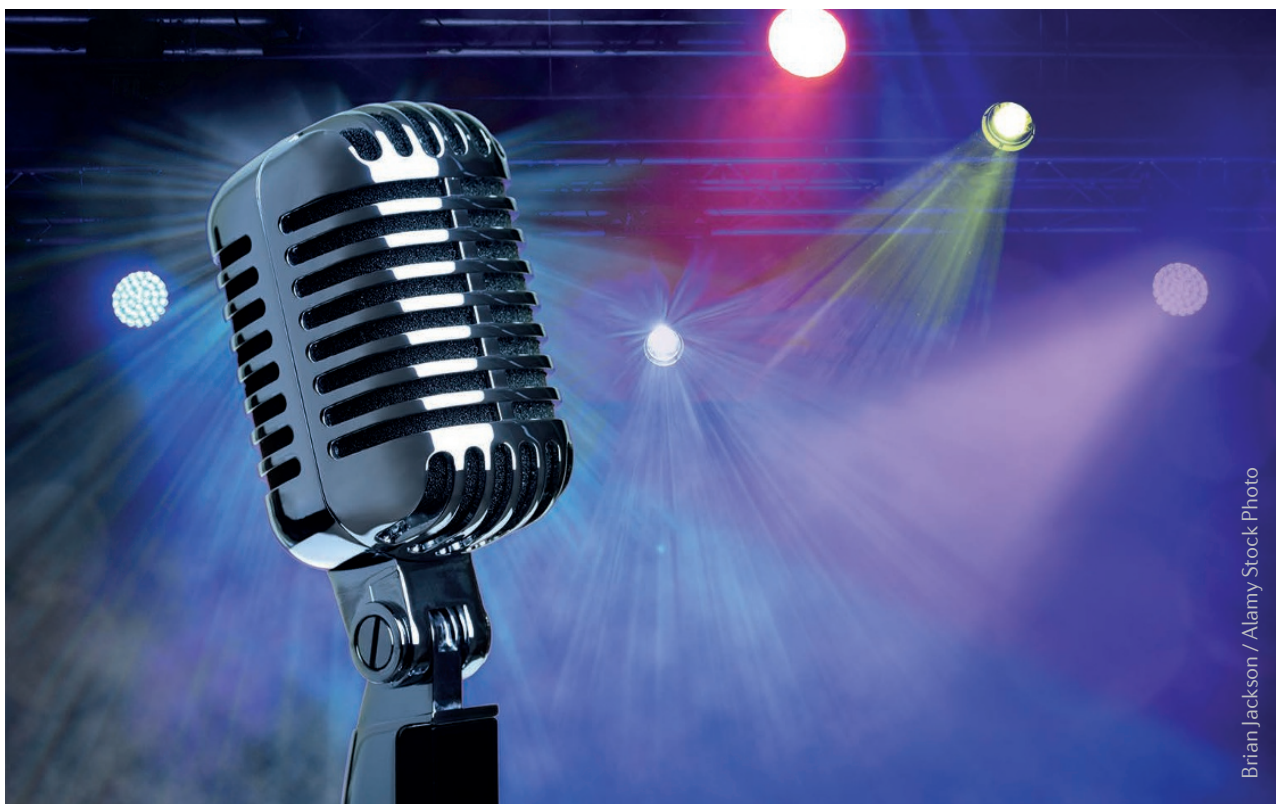

$\Rightarrow$ MILESTONE 9

\title{
Ximelagatran sets the stage for NOACs
}

For $>50$ years, vitamin $\mathrm{K}$ antagonists (MILESTONE 2) were the mainstay of oral anticoagulant therapy. In 2003, ximelagatran, an oral direct thrombin inhibitor (DTI), was approved in Europe for short-term venous thromboembolism (VTE) prophylaxis following orthopaedic surgery. The drug was subsequently withdrawn, and its application to the FDA was rejected, because of elevated levels of liver enzymes observed in treated patients.

However, the development of ximelagatran provided the proof of principle that a specific oral thrombin inhibitor could be effective in treating thrombotic disorders without the need for coagulation monitoring, and elucidated important characteristics of oral anticoagulants.

Thrombin is the final mediator of fibrin formation. Thrombin cleaves fibrinogen to fibrin: crosslinking of fibrin establishes the framework for a thrombus. Thrombin is, therefore, a logical candidate target for anticoagulant therapies.

In the mid-1980s, a team at AstraZeneca initiated a project to identify oral DTIs. Starting with small peptides of 2-5 amino acids based on known competitive inhibitors of thrombin, they developed their first clinical candidate, inogatran.
Although injected inogatran had anticoagulant activity and favourable pharmacokinetic properties in humans, oral bioavailability was $<5 \%$.

At this time, computer modelling was an emerging technology. Using thrombin structures from nuclear magnetic resonance studies, along with energy calculations for interactions between thrombin and potential inhibitors, they ultimately produced melagatran. This molecule was not only more potent than inogatran, but was also $<500 \mathrm{Da}$, which the team thought crucial to oral bioavailability (later confirmed by Lipinski's famous 'rule of five'). Unfortunately, although the oral bioavailability of melagatran was $>50 \%$ in dogs, it was only about $3-7 \%$ in humans.

Melagatran has three charged groups, and so this compound would be unlikely to cross the mucosal barrier in the intestine. The researchers, therefore, sought to generate a lipophilic prodrug that would be converted to melagatran after absorption. To do so, they introduced a new model for gastrointestinal permeability - the flux of the compound across a monolayer of colon cancer cell line cultured on plates with polycarbonate filters - that, unlike the rat intestinal membranes that had been used previously, did not metabolize the tested compounds. Using this model, they identified ximelagatran, a derivative of melagatran that could efficiently cross the intestinal barrier and be converted to melagatran after absorption.

Ximelagatran was investigated in numerous clinical trials for indications including the prevention of VTE after orthopaedic surgery, treatment of symptomatic VTE, stroke prevention in patients with atrial fibrillation, and secondary prevention of major cardiovascular events after myocardial infarction. In many of these trials, ximelagatran was efficacious. For example, in the double-blind, multicentre THRIVE III trial, ximelagatran was superior to placebo for the extended prevention of VTE. In the randomized, double-blind EXULT A trial, ximelagatran was superior to warfarin for the prevention of VTE in patients undergoing total knee replacement. These results formed the basis for the approval of ximelagatran in Europe.

Despite these improvements in primary end points, $\sim 6 \%$ of patients in most of the trials developed transient, asymptomatic elevations in levels of liver transaminases through unknown mechanisms. Owing to this observation, ximelagatran was withdrawn from the market, and did not receive approval in the USA. In a case-control study, these hepatic events were associated with particular alleles of the major histocompatibility complex, suggesting they were part of an allergic response.

The development of ximelagatran, however, laid the groundwork for the development of the non-vitamin $\mathrm{K}$ antagonist oral anticoagulants (NOACs) (MILESTONE 10). The tools used to generate ximelagatran most notably computer modelling and the intestinal absorption model - were important advances in drug development.

Megan Cully, Senior Editor, Nature Reviews Drug Discovery

\footnotetext{
ORIGINAL ARTICLES Eriksson, H. et al. A randomized, controlled, dose-guiding study of the oral direct thrombin inhibitor ximelagatran compared with standard therapy for the treatment of acute deep vein thrombosis: THRIVE I.J. Thromb. Haemost. 1, 41-47 (2003) | Francis, C. W. et al. Comparison of ximelagatran with warfarin for the prevention of venous thromboembolism after total knee replacement. N. Engl.J. Med. 349, 1703-1712 (2003) |Schulman, S. et al. Secondary prevention of venous thromboembolism with the oral direct thrombin inhibitor ximelagatran. N. Engl.J. Med. 349, 1713-1721 (2003)

FURTHER READING Gustafsson, D. et al. A new oral anticoagulant: the 50-year challenge. Nat. Rev. Drug Discov. 3, 649-659 (2004)
} 